\title{
Enantioselective Cu-Catalyzed Arylation of Secondary Phosphine Oxides with Diaryliodonium Salts toward the Synthesis of P-Chiral Phosphines
}

\author{
Rodolphe Beaud, Robert J. Phipps, and Matthew J. Gaunt* \\ Department of Chemistry, University of Cambridge, Lensfield Road, Cambridge CB2 1EW, United Kingdom
}

Supporting Information

ABSTRACT: Catalytic synthesis of nonracemic P-chiral phosphine derivatives remains a significant challenge. Here we report $\mathrm{Cu}$-catalyzed enantioselective arylation of secondary phosphine oxides with diaryliodonium salts for the synthesis of tertiary phosphine oxides with high enantiomeric excess. The new process is demonstrated on a wide range of substrates and leads to products that are well-established P-chiral catalysts and ligands.

$\mathrm{C}$ hiral phosphines are essential ligands for enantioselective metal-catalyzed reactions. ${ }^{1}$ Most of the chiral phosphines commonly used today display planar or point chirality where the asymmetric feature is presented in the carbon framework of the ligand. Although P-stereogenic phosphines have been known as effective ligands for enantioselective catalysis for over 40 years, ${ }^{2}$ they are less frequently utilized compared with more readily available planar- and point-chiral phosphines. Classical methods for the synthesis of P-stereogenic phosphines usually rely on auxiliary-based or resolution processes. ${ }^{3}$ Recently, however, advances in asymmetric catalysis have provided alternative methods to access these chiral phosphorus compounds via methods based on alkene phosphination, ${ }^{4 a}$ arylation $^{4 c}$ or alkylation $^{4 \mathrm{~d}}$ of secondary phosphines, and $\mathrm{C}\left(\mathrm{sp}^{2}\right)-\mathrm{H}$ activation of aryl phosphine derivatives. ${ }^{4-h}$ Despite these advances, the development of distinct strategies that provide convenient access to a broad range of $\mathrm{P}$-stereogenic phosphines remains an ongoing challenge in chemical synthesis. Herein we report a new method to form P-stereogenic organophosphorous compounds via enantioselective $\mathrm{Cu}$-catalyzed arylation of secondary phosphine oxides (SPOs) using diaryliodonium salts (DAISs).

Over the past 8 years, our laboratory has developed a new reactivity platform based on the combination of DAISs and $\mathrm{Cu}$ catalysts to generate an aromatic electrophile equivalent in the form of a putative $\mathrm{Cu}(\mathrm{III})$-aryl intermediate (Scheme 1a). ${ }^{5}$ As a result, the arylation of carbon-centered neutral nucleophiles, including arenes, ${ }^{5 \mathrm{a}, \mathrm{b}}$ alkenes, ${ }^{5 \mathrm{c}}$ and alkynes, ${ }^{5 \mathrm{~d}}$ has led to the realization of many distinct transformations. Furthermore, we and others have translated this novel activation mode to asymmetric catalysis using chiral bisoxazoline ligands to achieve the enantioselective C-arylation of enol silanes, ${ }^{6 a, b}$ allylic amides, $^{6 \mathrm{c}}$ and tryptamine derivatives. ${ }^{6 \mathrm{~d}, \mathrm{e}}$ In further expanding the scope of this asymmetric arylation platform, we questioned whether certain heteroatom nucleophiles could engage the catalytically generated aromatic electrophile to form configurationally stable products. To test this, we selected SPOs as suitable
Scheme 1. New Synthesis of P-Chiral Phosphines

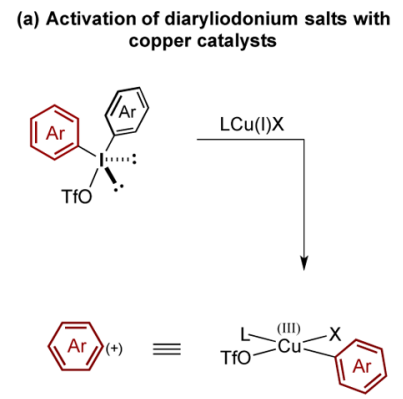

(b) 2'- phosphine oxide (SPO) to phosphinous acid tautomerization

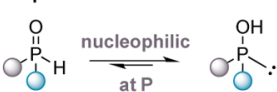

(c) enantioselective arylation of SPOs

catalytically generated aromatic electrophile

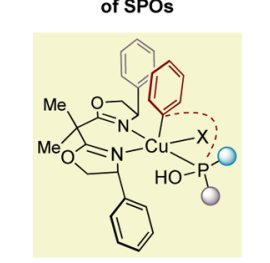

(d) copper-catalyzed enantioselective arylation of SPOs to $P$-chiral phosphines

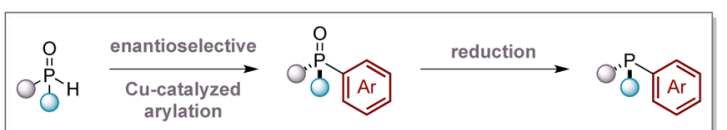

nucleophiles, whose arylation would lead to tertiary phosphine oxides (TPOs), precursors to P-chiral phosphines as well as useful asymmetric catalysts in their own right.

An SPO exists in equilibrium with its phosphinous acid form when in solution, with the latter recognized as a competent ligand for a range of transition metals (Scheme $1 \mathrm{~b}) .^{7}$ Therefore, we envisioned that a simple SPO would bind to a high-oxidationstate chiral $\mathrm{Cu}(\mathrm{III})$-aryl complex, formed through the action of a DAIS on the starting Cu catalyst, through the $\mathrm{P}$ atom, resulting in a square-pyramidal complex (Scheme 1c). The aromatic group would be transferred to the substrate as part of a stereocontrolled reductive elimination process to form the enantioenriched TPO. At the outset of our studies, we were aware of a report by Zhao that racemic arylation of SPOs can be achieved with diaryliodium salts and simple $\mathrm{Cu}$ catalysts. $^{8}$ By applying the knowledge accrued from our enantioselective arylation studies with $C_{2}$ symmetric bisoxazoline ligands, we began our studies with the straightforward merger of these sets of reaction conditions toward the development of a catalytic enantioselective arylation process (Scheme 1d).

We began by attempting the asymmetric arylation of SPO 1a with substituted DAIS 2a using $(S, S)$-diphenylbisoxazoline $\mathbf{4 a}$ as the chiral ligand, copper(II) triflate as the catalyst, and $\mathrm{Et}_{3} \mathrm{~N}$ as the base in $\mathrm{CH}_{2} \mathrm{Cl}_{2}$ solution at room temperature (Table 1).

Received: September 5, 2016

Published: September 30, 2016 
Table 1. Optimization of Enantioselective Arylation

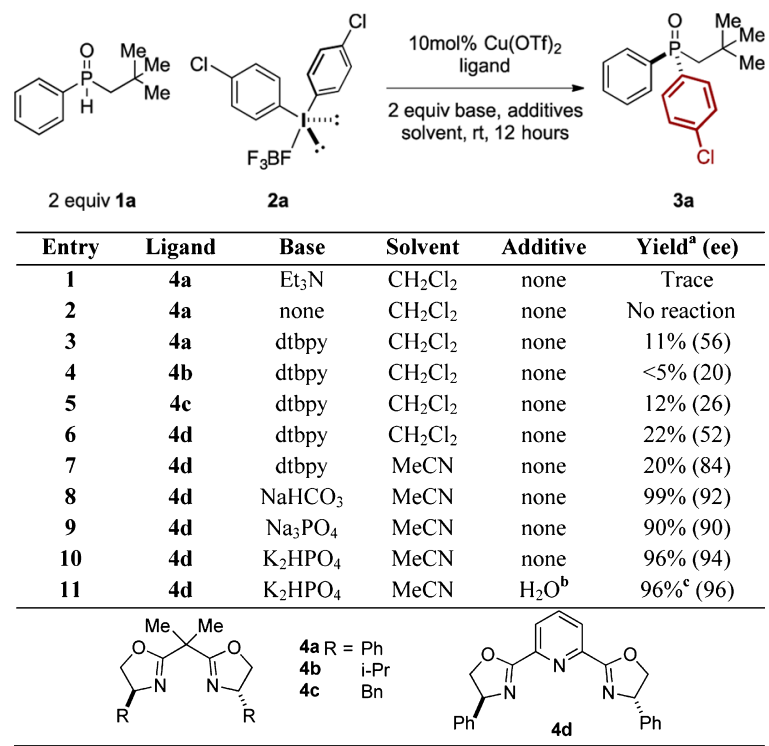

${ }^{a}$ Yields based on ${ }^{1} \mathrm{H}$ NMR analysis using 1,2-DME as an internal standard. ${ }^{b} 2$ equiv was added. ${ }^{c}$ Average yield over 3 runs.

Unfortunately, only traces of $\mathbf{3 a}$ were observed under these conditions (entry 1). However, we found that using the hindered base di-tert-butylpyridine (dtbpy) afforded the arylated TPO 3a with low conversion but importantly with modest enantiomeric excess (56\% ee) (entry 3 ). A survey of readily available ligands showed that changing substituents on the oxazoline moieties or using the corresponding tridentate pyridinebisoxazoline (pybox) catalysts led to no improvement using dtbpy as the base (entries 4 and 5). However, we were delighted to find that changing the solvent to $\mathrm{MeCN}$ while retaining $(S, S)$-diphenylpybox $4 \mathrm{~d}$ as the ligand and dtbpy as the base resulted in a dramatic increase in the ee of 3a, but only a modest yield. Moreover, changing the base to $\mathrm{NaHCO}_{3}$ resulted in a significant increase in conversion, with 3a isolated in $99 \%$ yield (with respect to DAIS 2a) and high ee. Further improvement was achieved by changing the base to $\mathrm{K}_{2} \mathrm{HPO}_{4}$, and we found that the presence of 2 equiv of water was important in securing a reproducible and robust reaction. Thus, the optimal conditions involved treatment of 2 equiv of SPO 1a with DAIS 2a as the limiting reagent in the presence of $10 \mathrm{~mol} \%$
$\mathrm{Cu}(\mathrm{OTf})_{2}, 12 \mathrm{~mol} \%$ pybox ligand $4 \mathrm{~d}, 2$ equiv of $\mathrm{K}_{2} \mathrm{HPO}_{4}$, and 2 equiv of water in $\mathrm{MeCN}$ with stirring for $12 \mathrm{~h}$ at room temperature, which gave 3a in $96 \%$ yield with $96 \%$ ee after purification by silica gel chromatography.

With an optimal process in hand, we next investigated the scope of the SPO component in this transformation (Table 2). Simple SPOs bearing methyl and $n$-alkyl substituents were arylated to give the desired TPOs $\mathbf{3 b}-\mathbf{d}$ with excellent ee and yield. SPOs with secondary branched alkyl groups also were excellent substrates for the arylation and gave high yields of $3 \mathbf{e}$ and $3 \mathbf{f}$ with excellent ee. Substrates with branching adjacent to the $\mathrm{P}$ atom gave mixed results. While arylation of $t$-Bu- and cyclohexyl-derived SPOs resulted in good yields of $3 \mathrm{~g}$ and $3 \mathrm{~h}$, respectively, the enantioselectivities were moderate. However, cyclopropyl- and isopropyl-substituted SPOs underwent arylation in excellent yields to give $3 \mathbf{i}$ and $3 \mathbf{j}$, respectively, with high ee using slightly modified conditions. More functionalized substituents, including benzyl and $\mathrm{CH}_{2} \mathrm{SiMe}_{3}$ groups, also gave the desired products $(3 \mathbf{k}, 3 \mathbf{3})$ in comparably high yields and ee.

We next investigated different DAISs displaying a variety of electronic and steric properties (Table 3). Electron-withdrawing groups at the para position were well-tolerated, and enantioenriched TPOs $3 \mathbf{m}-\mathbf{o}$ were obtained in excellent yields and ee. Halogenated arenes were also transferred smoothly to afford $3 p$ and $3 \mathbf{q}$ in good yields and enantioselectivities. Finally, more electron-rich aryls delivered $3 \mathbf{r}$ and $3 \mathbf{s}$ with remarkably high enantiomeric excess. After recrystallization, $3 \mathbf{r}$ was obtained as a single enantiomer, and its X-ray structure confirmed the absolute stereochemistry of the enantioenriched TPOs. ${ }^{9}$ DAISs displaying $p$-OMe or $p-\mathrm{NO}_{2}$ substituents were unsuccessful in this reaction. However, we found that other substituted arenes are transferred effectively; meta-substituted DIASs are effective in this process. For example, electron-deficient arenes $(3 \mathbf{v}-\mathbf{x})$, halogenated arenes $(\mathbf{3 y}, \mathbf{3 z})$, and simple alkyl-substituted arenes (3ab, 3ac) were transferred to form TPOs in excellent yields and ee. Conducting the reaction with disubstituted DAISs also led to the formation of the arylated TPOs 3ad-af with enantioselectivities of over $90 \%$ ee in excellent yields.

Finally, we sought to access P-stereogenic compounds bearing three groups of differing steric properties, namely alkyl, aryl, and ortho-substituted aryl. To achieve this goal, we reacted SPO $\mathbf{1 b}$ with ortho-substituted DAISs. The reaction occurred smoothly with $o$-trifluoromethoxy iodonium salt to form the electron-

Table 2. Enantioselective Arylation of SPOs: Scope of the SPO Component

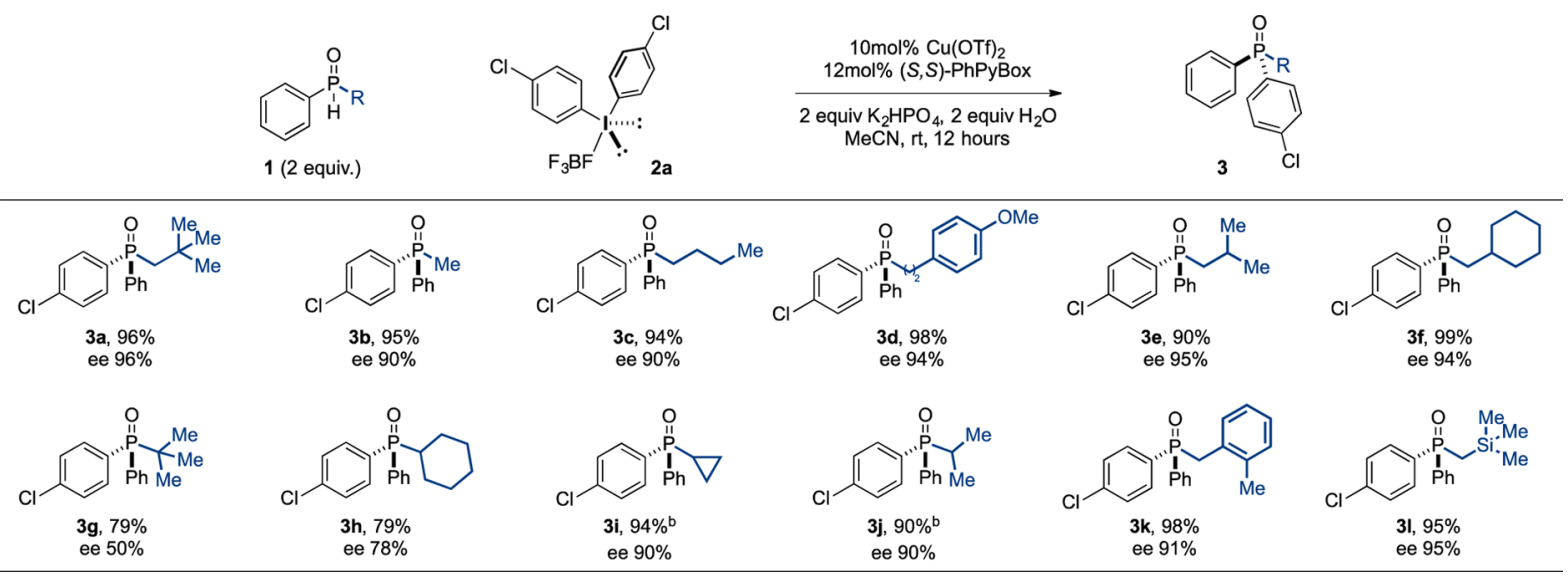

${ }^{a}$ Yields of isolated products based on $\mathbf{2 a}$ as the limiting reagent are shown. ${ }^{b}$ Reaction with degassed MeCN. 
Table 3. Enantioselective Arylation of SPOs: Scope of Aryl Transfer

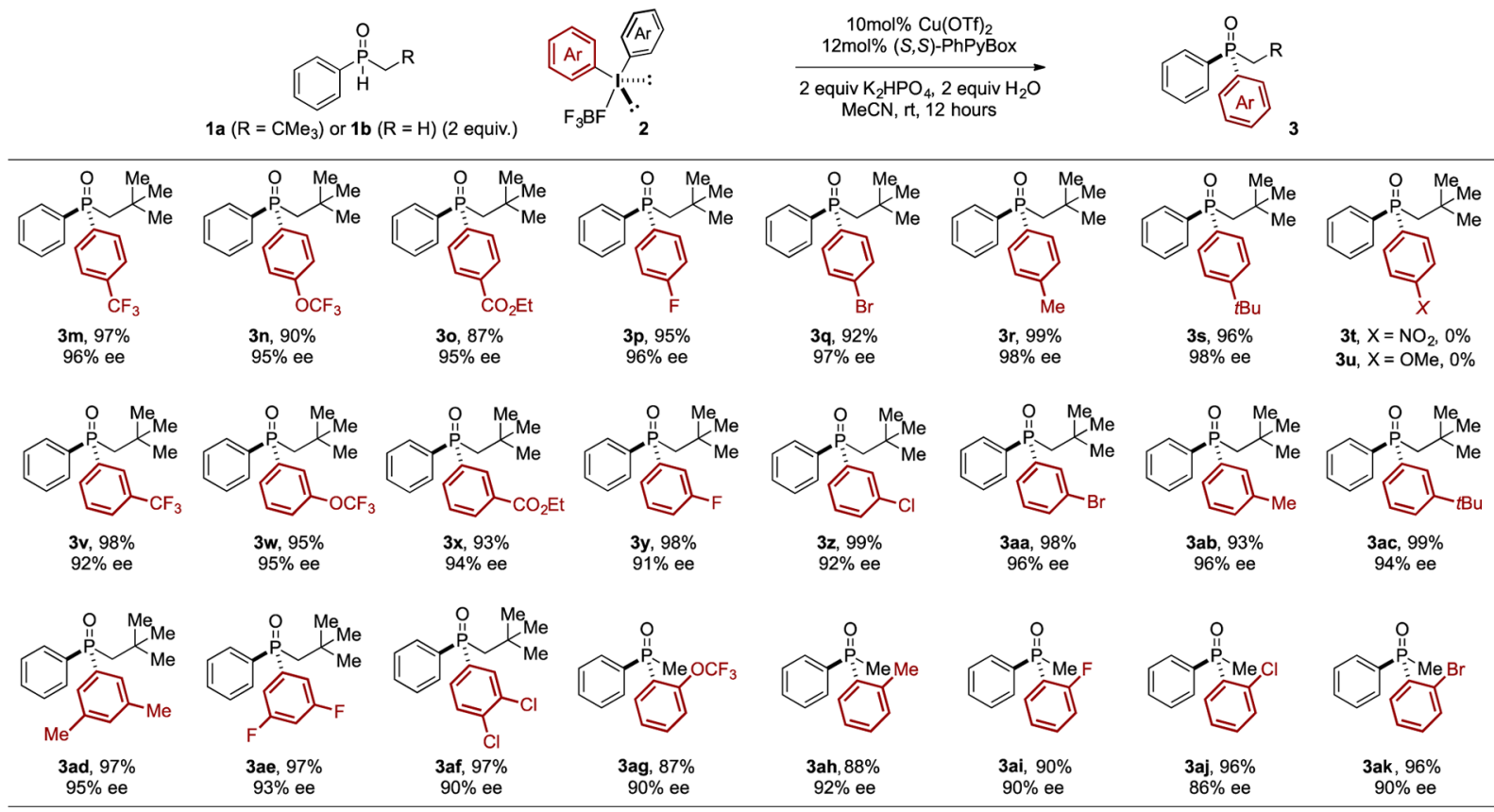

${ }^{a}$ Yields of isolated products based on $\mathbf{2}$ as the limiting reagent are shown.

deficient PAMP ${ }^{10}$ analogue 3 ag in excellent yield with very good ee. An $o$-tolyl iodonium salt reacted in almost quantitative yield to afford 3ah, again with excellent ee. We were also pleased to find that ortho-halogenated iodonium salts reacted smoothly to produce TPOs with a convenient handle for further functionalization (3ai-3ak) in high yields and ee.

Reflecting on a possible mechanism for this enantioselective arylation process, our first impressions were that the reaction would operate as an arylative kinetic resolution process. On the basis of the formation of TPO 3a, its enantioselectivity, and the assumption of first-order kinetics, this arylative kinetic resolution displays a selectivity factor of 148 . Although in principle the starting material should be produced with enantiomeric excess, we found that isolating the remaining starting material was not trivial. A deleterious oxidation process transforms the remaining SPO into the corresponding phosphinic acid derivative, which undergoes $\mathrm{O}$-arylation to give the phosphinate. However, it was possible to isolate the small quantities of the remaining SPO, and we were surprised that this material was either racemic or displayed very low enantiomeric excess and hence was not consistent with a classical kinetic resolution. To probe this unusual finding, we conducted a series of control reactions. We tested the stability of enantioenriched SPO (-)-1c under a number of conditions related to the optimal process and were surprised to find that in all cases the remaining SPO was partially or fully racemized in the presence of any of the reaction components (Scheme 2a) and accompanied by significant amount of the corresponding of aryl phosphinate. While this configurational instability of the SPO is contrary to most reports in the literature, we believe that racemization is the result of acid generated as a result of the deleterious oxidation pathway.

We also showed that the reaction of enantioenriched (-)-1c under standard conditions but without the chiral ligand gave a stereospecific arylation in moderate yield accompanied by oxidative arylation to racemic phosphinate 5a (Scheme $2 b$ ). Reaction of $(-)-\mathbf{1 c}$ with $(S, S)-4 \mathbf{d} \cdot \mathrm{Cu}(\mathrm{OTf})_{2}$ leads to the productive, matched formation of the arylation product in high
Scheme 2. Control Experiments

(a)
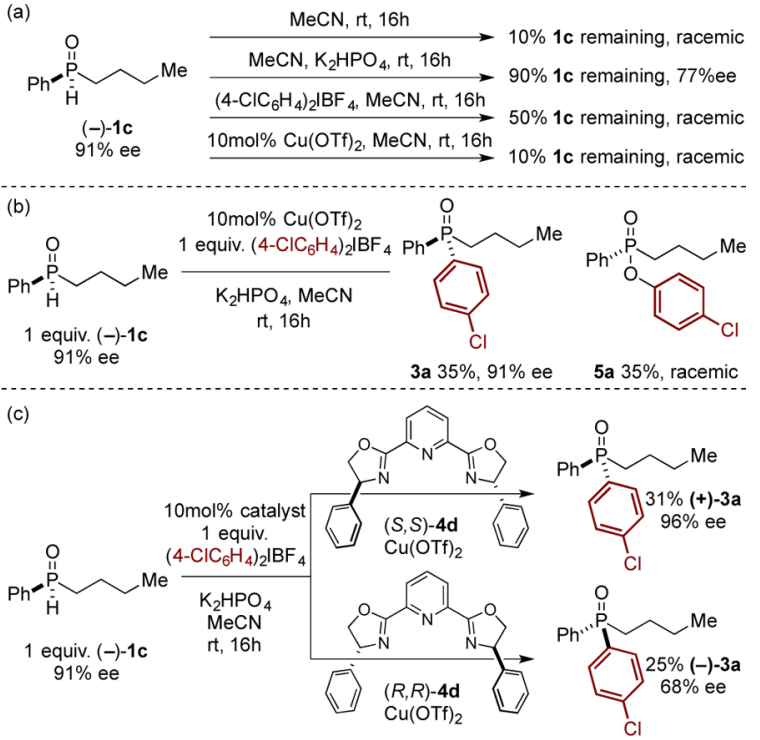

er. However, we were surprised to find that the supposed mismatched experiment (with $(R, R)-4 \mathbf{d} \cdot \mathrm{Cu}(\mathrm{OTf})_{2}$ ) gave a similar yield and high er of the opposite enantiomer of the product, accompanied by the corresponding arylated phosphinate in low yield and enantioselectivity (Scheme 2c). We rationalize this observation on the basis that the starting enantioenriched SPO must be racemizing during the reaction ${ }^{11}$ to form quantities of the enantiomer that is matched to the catalyst being used, resulting in arylation to give the observed TPO enantiomer. Taken together, these experiments hinted at the opportunity for a dynamic kinetic resolution arylation of a racemic SPO, but all attempts to secure such a transformation have failed; clearly, the mechanism of this enantioselective arylation process is more complex than simply a kinetic resolution. Despite this, it is clear that the distinct $\mathrm{Cu}$-catalyzed 
arylation process provides an effective way to produce enantioenriched TPOs in excellent yield with very high enantiomeric excess.

We demonstrated the utility of these enantioenriched TPOs through further transformations of the P-chiral scaffold (Scheme 3). We carried out Suzuki cross-coupling of 3ak to form a chiral

\section{Scheme 3. Transformations of TPOs}

(a) Cross coupling to enantioenriched, hindered TPOs
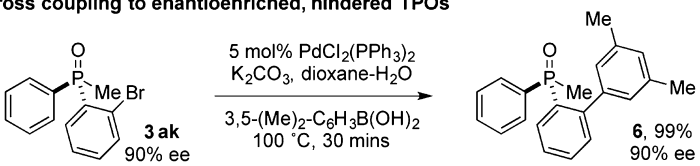

(b) Reduction of TPOs to $P$-chiral phosphine derivatives

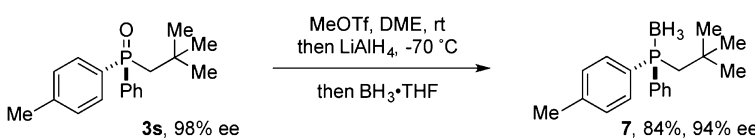

phosphine oxide analogue of the S/X-Phos-type ligands that are ubiquitous in Pd-catalyzed transformations. ${ }^{12}$ Although P-chiral TPOs are also effective ligands in a range of asymmetric reactions, ${ }^{13}$ the corresponding phosphines are more widely used in chemical synthesis. Therefore, we were pleased to find that reduction to the corresponding P-chiral phosphines can be achieved by treating the enantioenriched TPOs with a combination of MeOTf and $\mathrm{LiAlH}_{4}$ to form the P-chiral phosphine $-\mathrm{BH}_{3}$ adducts, providing potential opportunities for asymmetric metal-catalyzed transformations.

In summary, we have described a general and highly enantioselective route to access P-chiral tertiary phosphine oxides via a $\mathrm{Cu}$-catalyzed arylation process using readily available diaryliodonium salts. Although its mechanism remains unclear, this arylation process provides rapid access to a range of P-chiral phosphines that have great potential as ligands in catalytic enantioselective processes. Current studies are focused on elucidating the mechanism of this reaction and exploring the applications of the new ligand scaffolds.

\section{ASSOCIATED CONTENT}

\section{S Supporting Information}

The Supporting Information is available free of charge on the ACS Publications website at DOI: 10.1021/jacs.6b09334.

Procedures and spectral data (PDF)

Crystallographic data for $3 \mathbf{r}(\mathrm{CIF})$

\section{AUTHOR INFORMATION}

\section{Corresponding Author}

*mjg32@cam.ac.uk

\section{Notes}

The authors declare no competing financial interest.

\section{ACKNOWLEDGMENTS}

We are grateful to the European Commission for a Marie Curie International Outgoing Fellowship (R.J.P.), the ERC (R.B.), the EPSRC, and the Royal Society (M.J.G.) for fellowships. Mass spectrometry data were acquired at the EPSRC UK National Mass Spectrometry Facility at Swansea University.

\section{REFERENCES}

(1) (a) Tang, W.; Zhang, X. Chem. Rev. 2003, 103, 3029. (b) Fernández-Pérez, H.; Etayo, P.; Panossian, A.; Vidal-Ferran, A.
Chem. Rev. 2011, 111, 2119. (c) Phosphorus(III) Ligands in Homogeneous Catalysis: Design and Synthesis; Kamer, P. C. J., van Leeuwen, P. W. N. M., Eds.; Wiley: Chichester, U.K., 2012. (d) Borner, A. Phosphorus Ligands in Asymmetric Catalysis; Wiley-VCH: Weinheim, 2008.

(2) (a) Horner, L.; Siegel, H.; Büthe, H. Angew. Chem., Int. Ed. Engl. 1968, 7, 942. (b) Knowles, W. S.; Sabacky, M. J. Chem. Commun. 1968, 22, 1445. (c) Vineyard, B. D.; Knowles, W. S.; Sabacky, M. J.; Bachman, G. L.; Weinkauff, D. J. J. Am. Chem. Soc. 1977, 99, 5946.

(3) For recent examples, see: (a) Han, Z. S.; Goyal, N.; Herbage, M. A.; Sieber, J. D.; Qu, B.; Xu, Y.; Li, Z.; Reeves, J. T.; Desrosiers, J.-N.; Ma, S.; Grinberg, N.; Lee, H.; Mangunuru, H. P. R.; Zhang, Y.; Krishnamurthy, D.; Lu, B. Z.; Song, J. J.; Wang, G.; Senanayake, C. H. J. Am. Chem. Soc. 2013, 135, 2474. (b) Rast, S.; Mohar, B.; Stephan, M. Org. Lett. 2014, 16 , 2688. (c) Sieber, J. D.; Chennamadhavuni, D.; Fandrick, K. R.; Qu, B.; Han, Z. S.; Savoie, J.; Ma, S.; Samankumara, L. P.; Grinberg, N.; Lee, H.; Song, J.J.; Senanayake, C. H. Org. Lett. 2014, 16, 5494. (d) For a general overview, see ref $1 \mathrm{c}$.

(4) (a) Kovacik, I.; Wicht, D. K.; Grewal, N. S.; Glueck, D. S.; Incarvito, C. D.; Guzei, I. A.; Rheingold, A. L. Organometallics 2000, 19, 950. (b) Huang, Z.; Huang, X.; Li, B.; Mou, C.; Yang, S.; Song, B.-A.; Chi, Y. R. J. Am. Chem. Soc. 2016, 138, 7524. (c) Chan, V. S.; Chiu, M.; Bergman, R. G.; Toste, F. D. J. Am. Chem. Soc. 2009, 131, 6021. (d) Korff, C.; Helmchen, G. Chem. Commun. 2004, 530. (e) Lin, Z.-Q.; Wang, W.-Z.; Yan, S.-B.; Duan, W.-L. Angew. Chem., Int. Ed. 2015, 54, 6265. (f) Du, Z.-J.; Guan, J.; Wu, G.-J.; Xu, P.; Gao, L.-X.; Han, F.-S. J. Am. Chem. Soc. 2015, 137, 632. (g) Liu, L.; Zhang, A.-A.; Wang, Y.; Zhang, F.; Zuo, Z.; Zhao, W.-X.; Feng, C.-L.; Ma, W. Org. Lett. 2015, 17, 2046. (h) Sun, Y.; Cramer, N. Angew. Chem., Int. Ed. 2016, DOI: $10.1002 /$ anie.201606637.

(5) (a) Phipps, R. J.; Grimster, N. P.; Gaunt, M. J. J. Am. Chem. Soc. 2008, 130, 8172. (b) Phipps, R. J.; Gaunt, M. J. Science 2009, 323, 1593. (c) Phipps, R. J.; McMurray, L.; Ritter, S.; Duong, H. A.; Gaunt, M. J. J. Am. Chem. Soc. 2012, 134, 10773. (d) Suero, M. G.; Bayle, E. D.; Collins, B. S. L.; Gaunt, M. J. J. Am. Chem. Soc. 2013, 135, 5332.

(6) (a) Bigot, A.; Williamson, A. E.; Gaunt, M. J. J. Am. Chem. Soc. 2011, 133, 13778. (b) Harvey, J. S.; Simonovich, S. P.; Jamison, C. R.; MacMillan, D. W. C. J. Am. Chem. Soc. 2011, 133, 13782. (c) Cahard, E.; Male, H. P. J.; Tissot, M.; Gaunt, M. J. J. Am. Chem. Soc. 2015, 137, 7986. (d) Zhu, S.; MacMillan, D. W. C. J. Am. Chem. Soc. 2012, 134, 10815. Also see: (e) Kieffer, M. E.; Chuang, K. V.; Reisman, S. E. J. Am. Chem. Soc. 2013, 135, 5557.

(7) Shaikh, T. M.; Weng, C.-M.; Hong, F.-E. Coord. Chem. Rev. 2012, 256, 771.

(8) Xu, J.; Zhang, P.; Gao, Y.; Chen, Y.; Tang, G.; Zhao, Y.J. Org. Chem. 2013, 78, 8176.

(9) The X-ray structure of $\mathbf{3 r}$ confirmed the absolute stereochemistry.

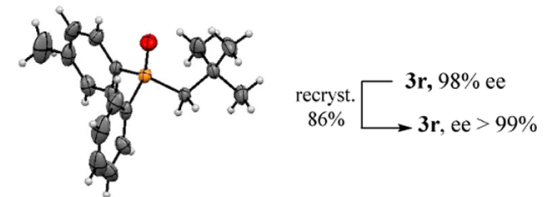

(10) (a) Vedejs, E.; Donde, Y. J. Am. Chem. Soc. 1997, 119, 9293. (b) Moncarz, J. R.; Brunker, T. J.; Jewett, J. C.; Orchowski, M.; Glueck, D. S.; Sommer, R. D.; Lam, K.-C.; Incarvito, C. D.; Concolino, T. E.; Ceccarelli, C.; Zakharov, L. N.; Rheingold, A. L. Organometallics 2003, 22, 3205. Also see ref $2 c$.

(11) A similar acid-promoted racemization has been reported. See: $\mathrm{Xu}$, Q.; Zhao, C.-Q.; Han, L.-B. J. Am. Chem. Soc. 2008, 130, 12648.

(12) (a) Surry, D. S.; Buchwald, S. L. Angew. Chem., Int. Ed. 2008, 47, 6338. (b) Martin, R.; Buchwald, S. L. Acc. Chem. Res. 2008, 41, 1461.

(13) Benaglia, M.; Rossi, S. Org. Biomol. Chem. 2010, 8, 3824. 